\title{
RESET
}

Recherches en sciences sociales sur Internet

4 | 2015

Les mondes du jeu

\section{Jouer avec l'échelle du monde : la pratique de World of Warcraft sur un serveur privé}

Changing the scale of the world. Playing World of Warcraft on a private server

\section{Vinciane Zabban}

\section{(2) OpenEdition}

\section{Journals}

Édition électronique

URL : http://journals.openedition.org/reset/464

DOI : $10.4000 /$ reset.464

ISSN : 2264-6221

\section{Éditeur}

Association Recherches en sciences sociales sur Internet

Référence électronique

Vinciane Zabban, « Jouer avec l'échelle du monde : la pratique de World of Warcraft sur un serveur privé », RESET [En ligne], 4 | 2015, mis en ligne le 21 décembre 2015, consulté le 14 novembre 2019. URL : http://journals.openedition.org/reset/464 ; DOI : 10.4000/reset.464

Ce document a été généré automatiquement le 14 novembre 2019.

(c) Association Recherches en sciences sociales sur Internet 


\title{
Jouer avec l'échelle du monde : la pratique de World of Warcraft sur un serveur privé
}

Changing the scale of the world. Playing World of Warcraft on a private server

\author{
Vinciane Zabban
}

\section{Introduction}

1 Les jeux vidéo émergent et se développent comme des produits à l'intersection des industries logicielles, culturelles et de loisir. Ils représentent aujourd'hui, avec les productions cinématographiques auxquelles ils sont assez étroitement articulés (Blanchet 2010), l'une des formes populaires les plus "globalisées» des industries culturelles et de la dite culture de masse soit parmi les produits culturels les plus répandus de manière standardisée à l'échelle mondiale. Les jeux en ligne apparaissent ainsi comme des objets particulièrement pertinents pour étudier l'impact social, culturel et technique des processus de globalisation et de massification informationnels et communicationnels contemporains. Lorsque ces jeux se développent sous la forme de "mondes", ils invitent d'autant plus les chercheurs à interroger ce caractère global et uniforme de leurs représentations et de leurs pratiques (Kline, Dyer-Witheford, and de Peuter 2003 ; Cassell and Jenkins 2000). A partir du milieu des années 2000, la notion de monde de jeu prend une ampleur particulière avec le développement à un niveau international de l'audience de jeux en ligne tels que World of Warcraft (Blizzard Entertainement, 2004). Les représentations communes, comme certains travaux de recherche orientés vers une analyse sémiologique de ces jeux, tendent en ce sens à présenter comme homogène la pratique de ces jeux - et en particulier celle des mondes jeux en ligne multijoueurs contemporains (Corneliussen and Rettberg 2008).

De fait, comme le souligne Erving Goffman, les espaces et pratiques de jeu supposent la création explicite de mondes d'objets et de sens relativement autonomes et distincts du monde ordinaire. Le fait de jouer ensemble implique de partager à un certain degré de 
cet univers de référence : on doit s'accorder par exemple sur la hierarchie de valeur accordée à une série de cartes à jouer. Mais à un certain degré seulement, puisque l'activité de jeu consiste elle-même en une négociation continue du sens de l'univers qui est mis en partage (notamment des exceptions à la règle), négociation qui autorise par des arrangements et ajustements locaux son investissement : toujours dans un jeu de cartes, l'inclusion ou l'exclusion de cartes spéciales, la modification des règles par exemple peut permettre d'ajuster la partie au nombre de joueurs présents, où à leur niveau d'experience (Goffman 1961). Sur ce point, il est intéressant de noter que la relation qu'ont les joueurs aux jeux vidéo, et en particulier aux jeux en ligne, a pu être par plusieurs aspects assimilée à celle de fans en ce qu'elle dévoile un rapport qui est jugé paradoxal à l'oeuvre originale. Cette relation particulière mêle dans le temps même désir d'appropriation et impératif de loyauté (Jenkins 2006). A propos des joueurs de jeu de rôle, Daniel Dayan avait d'ailleurs soufflé à Gary Alan Fine l'expression de copyrighted subculture pour qualifier la nécessité qu'ont les joueurs de jeux de rôle de recourir en cas de doute ou de conflit d'interprétation à l'arbitrage d'une autorité centrale, c'est-à-dire de ses auteurs (Dayan 1986).

Or la question que nous nous posons ici concerne précisément les pratiques du jeu qui se développent a priori en dehors de l'influence de cette autorité. Certains de ces jeux en ligne joueurs semblent en effet vouloir faire "bande à part" en investissant des serveurs dits "privés", c'est à dire des versions du jeu qui sont déployées et maintenues illégalement hors du contrôle de l'éditeur. On pourrait aisément faire l'hypothèse que les serveurs privés représentent des réponses à ce contexte de globalisation et de standardisation culturelle des mondes de jeu, en constituant des espaces alternatifs voire critiques et contestaires de «lectures» du monde de jeu. Techniquement, les serveurs privés peuvent en effet proposer des transformations en profondeur de l'univers de jeu, et correspondre à une lecture oppositionnelle davantage que négociée du jeu, au sens de Stuart Hall (1994). Nous verrons ici que l'observation que nous avons réalisée de manière exploratoire va plutôt à l'encontre de cette idée. A l'instar d'autres travaux de recherches récents en sociologie (Berry 2012 ; Coavoux 2010b), nous avons en effet pu montrer que les joueurs de jeux en ligne se présentaient hétérogènes en profil comme en pratique, et que s'il n'y avait qu'un monde de jeu à l'échelle globale à partager, il restait de nombreux espaces de négociation collective et individuelle et d'ajustement locaux pour la pratique. Autrement dit, qu'à la nécessité d'une régulation sociale et technique globale, c'est-à-fire centralisée et applicable à tous, correspondait la nécessité d'un certain nombre d'espace pour le développement d'ajustements et de négociations locales (Zabban 2009 ; Zabban 2011a). Or la pratique des serveurs privés que nous avons observée nous parait aller plutôt dans ce sens, en proposant, davantage qu'une interprétation nouvelle du jeu, la création de conditions d'émergence d'un entre soi pour les joueurs. Cet article propose donc, partant de l'analyse de ces formes particulières de la pratique de jeux en ligne, d'ouvrir une réflexion plus générale sur la façon dont sont articulées échelles locales et globales dans ce contexte numérique.

Nous ferons pour cela un bref retour sur la façon dont les jeux en ligne se sont développés parallèmement à la croissance de leur audience, puis nous présenterons le contexte spécifique du jeu World of Warcraft, avant de nous intéresser enfin à la manière dont la pratique des serveurs privés dévoile ici un goût chez les joueurs pour une certaine configuration de sociabilité ludique, autrement dit leur autorise, plus qu'une lecture alternative du jeu et de son univers, un jeu avec l'échelle du monde. 
Cette réflexion prend appui sur un terrain exploratoire concernant la pratique du jeu World of Warcraft sur des serveurs privés par entretiens en face à face et en jeu, et par observations en jeu. Ce terrain est abordé avec l'éclairage apporté par la comparaison avec une analyse plus complète qui a été menée via une observation participante menée pendant 4 ans sur un serveur officiel sur la version officielle de ce jeu dans le cadre d'une thèse menée sur la conception et la pratique des mondes de jeu en ligne (Zabban 2011b).

\section{Mondes fictionnels et émergence de services ludiques à une échelle globale}

D'abord restreints à des communautés spécifiques et notamment universitaires, les univers de jeu en ligne s'étendent progressivement à partir de la fin des années 1990, parallèlement à la diffusion de l'usage d'Internet. Se développe alors en Occident le genre nommé « Massive Multiplayer Online Role Playing Games » : jeux de rôle en ligne massivement multijoueurs. Ces jeux, qui s'inscrivent dans la continuité des MUDs, des jeux de rôle et des jeux vidéo d'aventure, proposent un service d'accès à un espace d'activités et de rencontres ludiques, ainsi que le maintien et le développement de cet espace de jeu. Ces nouveaux "mondes" de jeu, tels que Ultima Online (Origin System 1997), Everquest (Sony Entertainement 1999), Dark Age of Camelot (EA mythic 2001)1, sont alors dits persistants, parce qu'ils sont accessibles aux joueurs en permanence via la connexion à un serveur de jeu, et ont une durée de vie assez longue. Le plus grand succès d'entre eux en occident est alors Everquest, sorti en 1999, qui comprendra jusqu'à 500.000 comptes abonnés. On pense alors qu'il s'agit d'un pallier d'audience maximal, qu'aucun titre ne pourra dépasser (Bartle 2004). Sur ces dispositifs de jeux, s'expérimentent, se développent, et se stabilisent des systèmes de jeu, d'interface et d'organisation de l'activité qui deviennent peu à peu des normes pour les joueurs comme pour les développeurs et sont reprises par les titres suivants.

Ces titres ne présentent pas tous exactement le même fonctionnement organisationnel et commercial, ce qui affecte la marge et les modalités de contrôle qui sont applicables au jeu et aux joueurs: l'éditeur en particulier peut entretenir une relation plus ou moins proche avec le dévelopeur.Par ailleurs, le degré d'autonomie déleguée à l'organisation chargée de la gestion du service est variable. La régulation ainsi que l'animation de ces jeux - qui justifie en partie le coût de l'abonnement - fait en effet appel à une équipe d'animation et de support, parfois rémunérée et parfois bénévole. Contrairement à World of Warcraft pour lequel Blizzard assume à la fois les fonctions d'éditeur et de développeur, dans le cas de DaoC par exemple, le développeur (EA Mythic) est distinct de l'éditeur (GOA pour l'Europe entre 2001 et 2009). GOA centralise entre autres choses l'animation du jeu. La distance avec le développeur ne facilite pas l'intervention sur les éléments techniques et structurels du jeu (résolution de failles, modifications et améliorations). En revanche, d'après les discours tenus par d'anciens membres de cette équipe d'animation et joueurs ${ }^{2}$, précisemment parce qu'elle est détachée des responsabilités et des contraintes de développement et se développe au niveau d'un service « local » avec en France quelques centaines de joueurs connectés en simultané sur un serveur, la régulation comme l'animation en jeu peuvent alors se dérouler de manière assez personnalisée. Cette configuration confère aux joueurs un 
important sentiment d'influence sur l'évolution de l'univers, ce qui fait alors partie du caractère exceptionnel de ce type d'expérience ludique.

Au tout début des années 2000, il est évident depuis longtemps que les services de jeux en ligne sont très rentables pour les opérateurs comme pour les éditeurs. ${ }^{3}$ L'engouement pour les entreprises en ligne, les capacités techniques et la progression de l'équipement constituent un contexte propice pour le développement de ces formes actualisées d'espaces cybernétiques. Dans le milieu des années 2000, deux d'entre elles vont marquer significativement et durablement les représenations des espaces et des modes d'interaction et de sociabilité en ligne : Second Life (2003), un espace de création de type expérimental mené dans une logique ouverte par Liden Lab (Boellstorff 2008 ; Malaby 2009) et World of Warcraft (Blizzard Entertainment, 2004). Le premier marque surtout les mondes médiatiques et académiques par sa dimension expérimentale, mais le second amène l'audience des jeux en ligne à une nouvelle échelle, en impactant la conception du jeu comme sa pratique.

\section{World of Warcraft : effets et dispositifs de régulation de la massification d'un jeu en ligne}

World of Warcraft et ses différentes extensions marquent une massification effective des usages de ce type de jeu. Si ses prédécesseurs avaient pu recueillir au maximum les suffrages de quelques centaines de milliers de joueurs en occident, et jusqu'à un à deux millions en Asie, WoW accueille en quelques mois plusieurs millions d'abonnés - et jusqu'à 11 millions en 2011. Répartis à l'internationale (Europe, Amérique, et plus progressivement Asie), ces millions de joueurs investissent en moyenne 20 heures par semaine à parcourir et à interagir dans le monde d'Azeroth (Williams, Yee, and Caplan 2008) pendant souvent plusieurs années. Ils forment des collectifs de joueurs (guildes), nouent des relations plus ou moins intenses et durables, investissent et construisent des espaces de communication en jeu comme hors jeu (Zabban 2009).

\section{Un monde de jeu commun}

D'un point de vue technique, ce monde de jeu est répliqué à l'identique sur différents serveurs, qui peuvent chacun accueillir simultanément jusqu'à 5000 connexions de joueurs. Blizzard, l'éditeur du jeu, contrôle l'accès à ses serveurs auxquels se connectent les logiciels clients des joueurs (moyennant l'équivalent de 13 euros par mois). Ainsi, quelque soit la localisation des joueurs, en théorie le monde d'Azeroth proposé par WoW reste le même - et il doit le rester pour que l'on puisse jouer ensemble, ou du moins en avoir le sentiment.

La lecture de l'espace du jeu comme un espace social a déjà fait l'objet d'analyse (Coavoux 2010b). Nous avons pu, pour notre part, montrer dans des travaux précédents comment au sein de cet espace social devaient cohabiter des joueurs aux profils et intérêts non seulement distincts mais parfois conflictuels. Il apparait ainsi que le partage de ces mondes de jeu n'est pas une évidence, mais est négocié à différentes étapes aussi bien de leur conception que de leur pratique. Dans le cadre de cette pratique il est apparu en outre que ces joueurs pouvaient mobiliser les espaces techniques et médiatiques hors jeu, dans le but de conférer davantage de légitimité à leur vision du monde (Zabban 2011a). 
12 Pour que l'on puisse tous jouer avec les mêmes règles, la définition d'un monde commun semble ainsi soumise à la nécessité d'une autorité centrale, d'un maître du jeu. Ce maître du jeu est également celui qui peut, en apportant des modifications substantielles au jeu, rebattre les cartes des positions obtenues dans cet espace social et donc potentiellement porter atteinte à la légitimité de certains acteurs de la communauté en altérant la valeur de leurs biens et compétences acquises, ainsi que l'ont montré les travaux de Samuel Coavoux (2010a). Car les manières de jouer sont non seulement hétérogènes, ce qu'a pu montrer pour sa part de manière détaillée Vincent Berry (2012), mais inégales: les règles du jeu leur confèrent de fait des valeurs différenciées. Les éditeurs ont donc un pouvoir indirect mais essentiel sur les usages du jeu via le contrôle qu'ils possèdent sur les règles : règles qui comportent des dimensions sociales, techniques et juridiques, cf. (Mauco 2011 ; De Paoli and Kerr 2012).

13 Si les activités créatrices des joueurs autour du jeu et leur participation très active à son évolution ont pu étonner les observateurs, la réponse à la question "à qui appartient le jeu? » posée par T.L. Taylor dans son ethnographie d'Everquest (Taylor 2006a) parait finalement étonnamment claire: ce qui fait le jeu dépend en grande partie des joueurs, mais le jeu appartient toujours in fine à ses éditeurs, et ce avec d'autant plus de pertinence que l'on assiste à une massification de ses usages. On constate ici un phénomène similaire à celui observé par Gary Alan Fine à propos du jeu de rôle Donjons et Dragons. Le sociologue décrit en effet le double mouvement de centralisation et de clarification des règles du jeu que connaît le titre - qui va lui-même ancrer ses références dans un univers fictionnel au succès populaire (l'heroïc fantasy, et essentiellement dans l'oeuvre de Tolkien, cf. au fur et à mesure de sa diffusion sur le territoire américain puis à l'internationale (Fine 2015). Fine souligne tout particulièrement l'effacement des adaptations et des ajustements locaux des mécanismes et arbitrages du jeu de rôle à l'occasion des parties organisées entre joueurs de différents horizons pendant les conventions. Il évoque à ce sujet des problématiques qui préfigurent celles des jeux multijoueurs en ligne, tels que les incertitudes sur les comportements des autres joueurs liés à une faible interconnaissance et à un engagement dans l'interaction variable, ainsi qu'à une hétérogénéité des profils sociaux et culturels des joueurs, à une diversité de pratiques et de représentations du jeu. Ces incertitudes se traduisent le plus souvent par le renforcement d'une régulation « par le jeu », qui clarifie le cadre d'action des joueurs et minimise les marges d'interprétation et de négociation possibles.

L'effet de la « massification » de l'audience des joueurs sur la production de ce type de jeu comme sur la composition et sur les pratiques des populations de joueurs est très important. Comme souligné précédemment, les premiers mondes de jeu en ligne accueillent quelques centaines de joueurs; à l'instar de nombreuses premières formes de collectifs médiatisés, ils sont à même d'offrir à leurs participants, par la diversité géographique et professionnelle des recrutements, un sentiment d'alterité, tout en conservant une relative homogénéité sociale, qui est souvent maintenue par des logiques de sélection élitaires ${ }^{4}$. Ce faible nombre de participants et la sélectivité sociale, technique et économique de leur recrutement favorisent le développement d'un degré relativement élevé d'interconnaissance, d'une forme d'entre-soi qui permet par exemple à des effets de contrôle par la réputation de se développer. Le déploiement dans ce contexte de dispositifs de régulation "par le haut» assez souples avec des équipes de maîtres du jeu aux profils sociaux eux-même proches de ceux des 
consommateurs, et qui disposent de marges d'action importantes, rend par ailleurs relativement peu coûteuse la gestion personnalisée, au cas par cas, des interactions et des conflits, ou même lorsque cela est techniquement possible, les modifications de l'environnement de jeu.

\section{Une infrastructure globale, des ajustements locaux}

Dès 2006, T.L. Taylor s'interroge sur les caractéristiques de cultures ludiques émergentes et insiste sur l'importance de la prise en compte de l'ancrage contextuel des pratiques de jeu, pour leur compréhension. Dans l'article «Does WoW change everything? How a PvP server, a multinational player base and surveillance mod scene caused me pause ", Taylor raconte son étonnement lorsqu'elle découvre des logiques d'interaction des joueurs sur un serveur anglophone européen de World of Warcraft, elle qui a conduit son ethnographie participante dans le monde d'Everquest et au sein des communautés nord américaines (Taylor 2006b). Elle pointe notamment l'importance prise sur ce serveur de WoW à la fois par l'appartenance nationale, l'âge et le développement de formes de surveillance et de stratification outillées ${ }^{5}$ et collectives, qu'elle n'a pas rencontrées dans ses expériences précédentes.

16 Avec la massification de l'audience des joueurs, deux questions, qui se présentent à notre sens en miroir l'une de l'autre, vont en effet émerger : celle de la standardisation des mécanismes et de l'environnement de jeu, et celle contraire de l'émergence " par le bas » de formes d'interaction et de régulations que certains chercheurs vont appeler des cultures du jeu.

17 Le modèle choisi par l'éditeur de WoW est en ce sens assez parlant : d'une part, Blizzard maintient un contrôle centralisé de l'accès à ce que, suivant Bowker et al (2009), on peut assimiler à une infrastructure informationnelle, et qui est temporairement stabilisée autour d'un serveur, de bases de données, d'un programme du jeu. L'éditeur offre et contrôle ainsi des dispositifs de jeu suffisamment génériques pour convenir à une large gamme de joueurs en assurant a priori une équité des conditions de jeu. D'un autre côté, Blizzard délègue aux collectifs et aux espaces sociaux et techniques qui se développent en ligne, la tâche de réaliser et de diffuser les codes et les outils de pratiques spécifiques et de trouver les supports pour des ajustements locaux (outils d'information, bases de données joueurs, aides, forums, addons, etc.). L'éditeur peut ainsi se désengager d'une partie de la gestion de l'hétérogénéité des usages de son produit, ce qui lui évite oûtre des frais supplémentaires de développement le risque d'être pris à parti dans l'une ou l'autre des formes émergentes de cultures du jeu. Ce modèle a déjà été observé dans le cas d'un jeu plus ancien par Manuel Boutet, qui montre la façon dont l'implication des joueurs dans des formes d'appropriation de l'espace technique du jeu constitue un " point de frottement » et rend visible aux uns et aux autres la diversité de leurs mondes sociaux (Boutet 2010). Malgré le fort développement d'un "métajeu» en ligne (forums, bases de données, sites d'informations), d'espaces sociaux dans lesquels certains acteurs prennent des positions de pouvoir importantes, et d'une économie, plus ou moins légale et parallèle au jeu (intermédiaires, entreprises de triche, "goldfarmer » cf. De Paoli et Kerr 2012), Blizzard garde dans cette configuration un contrôle important sur l'évolution du jeu. 


\section{Serveurs privés et jeu officiel : des interprétations alternatives du monde de jeu?}

18 configuration et offrent une perspective intéressante pour questionner plus en avant la façon dont les jeux en ligne ont vu émerger des solutions à l'articulation de cultures globales et locales. Nous nous penchons donc particulièrement ici sur le cas des pratiques de jeu qui cherchent volontairement à rester à l'écart de l'oeuvre officielle en pratiquant le jeu sur des copies illégales et - techniquement parlant - locales de ce dernier, ou serveurs dits " privés " (Debeauvais et Nardi 2010). Comme nous le verrons, les serveurs privés ne modifient pas pour l'essentiel ou de manière radicale le monde de jeu - ils sont et restent des copies du jeu officiel, et en conservent l'infrastructure. Ils modifient en revanche l'échelle et les conditions de sa pratique, en n'accueillant pour la plupart qu'une ou plusieurs centaines de participants - contre quelques milliers sur les serveurs officiels, et en déplaçant de fait les modes de régulation du jeu. au déploiement du jeu. On trouve en ligne des traces et des récits montrant que de manière très précoce, avant même la sortie commerciale de WoW, soit pendant les phases de test réservées à un nombre limité de joueurs, des solutions techniques sont recherchées pour jouer au jeu en se passant de la médiation de l'éditeur. Parallèlement aux derniers réglages du jeu et à la construction progressive de son public, des communautés de développeurs s'organisent déjà autour de la création par ingénierie inverse d'émulateurs qui permettent de mettre en ligne un serveur autonome de jeu. ${ }^{6}$ Ces émulateurs sont ensuite diffusés et mis à disposition d'individus qui souhaitent déployer des serveurs privés. Les administrateurs de serveurs privés présentent des compétences techniques inégalement développées : la plupart se contentent de copier le code et les scripts existants et disponibles alors que d'autres interviennent de manière plus consistante dans le déploiement du serveur et des programmes de jeu pour les modifier.

De nombreux serveurs privés de WoW voient le jour de cette manière depuis 2003/2004 et sont maintenus pour des durées plus ou moins pérennes. Le déploiement de ces serveurs est de droit illégal, mais de fait assez largement toléré par l'éditeur. Les travaux conduits par Bruno Vétel sur les serveurs privés d'un jeu en ligne développé par une entreprise française montrent en effet que pratiques des serveurs privés et officiels ne sont pas concurrentielles : les joueurs qu'il interroge utilisent par exemple les serveurs privés pour faire des expériences particulières en jeu qui n'ont pas d'impact sur leurs comptes officiels (choix de progression des personnages, effets de réputation, triche, etc.). Et lorsque l'éditeur réagit, c'est surtout en se retournant contre un nombre restreint de ces acteurs à la marge, c'est-à-dire les plus attractifs et ceux ont des visées commerciales évidentes par une multiplication de leur offre de services sur le modèle $d u$ "free-to-play" (accès gratuit au jeu mais vente de personnages, d'objets, etc. qui facilitent la progression) (Vétel 2013). Dans le cas de WoW les administrateurs de serveurs privés sont ainsi peu inquiétés, et leurs activités sont assez largement publicisées. Les joueurs qui n'hésitent pas à investir leur temps, et parfois leurs économies dans ces espaces dont la pérennité est a a priori incertaine ont en fait plus à craindre leur disparition du fait du désengagement des administrateurs, que du fait de la fermeture par voie légale de ces copies illégitimes de jeu. Ce risque est

RESET, 4 | 2015 
néanmoins limité par la multiplicité des serveurs privés et par la mise en place de nombreux dispositifs qui facilitent la circulation des joueurs entre ceux-ci : vente des bases de données joueurs à un concurrent en cas d'abandon, offres de copies des personnages d'un compte d'un serveur à un autre, moyennant des conditions plus ou moins avantageuses, etc. Les migrations d'un serveur privé à un autre paraissent ainsi courantes, ce qui a été confirmé lors des entretiens conduits avec deux joueurs du serveur privé que nous avons plus particulièrement observé. Suite à un désaccord avec les équipes de gestion, ceux-ci ont en effet organisé la migration partielle de leur guilde sur un autre serveur privé. En effet les serveurs privés observés n'apparaissent pas comme des copies de WoW qui seraient isolées les unes des autres, mais forment au contraire une sorte de réseau d'espaces de jeu reliés entre eux. Au moment de l'observation en 2013, des portails (tels que Gowonda, ou RPG Fantasy en France) recensent entre 100 et 200 serveurs francophones et en proposent un classement qui est fondée sur les votes des joueurs.

21 Partant de ces portails, nous avons pu constater que ces différents serveurs mettaient en place des supports médiatiques relativement identiques. Le serveur est en effet généralement associé étroitement à un forum de discussion comprenant des informations actualisées concernant le nombre de joueurs connectés, mais aussi à des canaux de discussion instantanée (IRC) ainsi qu'à un serveur de discussion vocale (TeamSpeak). Ces serveurs en concurrence pour une audience, accueillent des populations de joueurs variables. Ainsi que souligné, et contrairement à ce qui aurait pu être attendu, la mise en place comme la pratique des serveurs privés ne semble pas majoritairement ressortir de ce qui aurait pu être un «décodage oppositionnel », au sens de Stuart Hall (1994) du produit officiel du jeu, c'est-à-dire d'une interprétation du monde de jeu qui se présenterait comme allant à l'encontre de celle «encodée » par l'éditeur. On aurait en effet pu imaginer le déploiement d'interprétations ou de pratiques alternatives via ces dispositifs qui autorisent théoriquement de véritables révolutions ou du moins des déviations conséquentes vis à vis de la version officielle du monde de Warcraft. On aurait pu également imaginer qu'a l'instar de phénomènes qui ont pu être observés sur les serveurs officiels (Rueff 2010 ; Shaw 2011), ces alternatives soient portées par des groupes sociaux singuliers. Mais, en résonnance avec ce que peut observer Olivier Caïra dans le déroulé des parties de jeu de rôle, s'il est de droit tout à fait possible que les joueurs optent pour des altérations majeures des univers de jeu, ce comportement est de fait tout à fait rare (Caira 2007). Le fait que nous ne l'ayions pas observé sur ce terrain exploratoire ne signifie bien entendu pas que type d'appropriation n'existe pas, mais il paraît plus "privé » que la plupart des cas que nous avons recensés, dans le sens où la proposition de tels types de serveurs n'apparait pas de manière évidente sur les espaces populaires d'information. Dans les cas observés, les serveurs privés peuvent être définis pour certains d'entre eux a maxima comme des lectures «négociées" de la version officielle du jeu? ${ }^{7}$. Ainsi en est-il par exemple des serveurs qui proposent l'accès à une version dite vanilla ${ }^{8}$ de WoW soit la version considérée comme originale (déployée de fin 2004 à 2007), qui précède la sortie de la première extension "Burning Crusade". Ces serveurs offrent ainsi aux joueurs la possibilité d'échapper à la fameuse "redistribution des cartes" opérée par l'éditeur.

Plusieurs éléments peuvent expliquer l'absence de modifications profondes opérées par les administrateurs de serveurs privés vis à vis des versions officielles. La dimension technique en est une. Pour des raisons qui paraissent étroitement liées à des questions de développement et de diffusion techniques, la plupart des serveurs privés proposent 
la même version du jeu, qui est antérieure à 2010. Ainsi que l'a montré Bruno Vétel (2013) des communautés de joueurs et d'animateurs s'organise autour de la mise en place de serveurs privés, mais les compétences en programmation et la maîtrise des outils techniques permettant de disposer d'une version du jeu relativement stable sont en fait l'apanage d'un petit nombre. Cette activité et les ressources qu'elle produit sont ainsi relativement centralisées et standardisées, autorisant de fait peu de possibilité de changements. Quant aux modifications exigeant des compétences informatiques moindres, touchant par exemple aux réglages des mécanismes de jeu, elles peuvent présenter à l'échelle d'un univers de jeu aussi complexe que celui de Blizzard des difficultés au moins similaires. Au final, comme Debauvais et Nardi le soulignent à propos de Ragnarok Online, les altérations de design qui sont principalement proposées par ces serveurs (progression facilitée, visibilité sur l'état du jeu, etc.) le sont essentiellement pour pallier la faiblesse des populations de joueurs qui les investissent. Le serveur que nous avons plus particulièrement observé se revendique quant à lui être « le seul serveur privé $100 \%$ Blizzlike » c'est-à-dire proposer une version du jeu qui soit la plus fidèle possible à la version officielle. L'observation en jeu et la comparaison avec la pratique des serveurs officiels montrent que ce positionnement n'est pas sans conséquence. En effet, l'investissement par une population de 100 à 200 joueurs de dispositifs de jeu prévus pour un millier d'entre eux limite notamment certaines facilités de progression (p.ex., via la difficulté à établir des relations de commerce en jeu équivalentes à celles d'un serveur officiel). Un autre problème qui se pose aux serveurs privés dans le cas de modifications substantielles est le manque de cohérence avec l'importante infrastructure informationnelle qui est alimentée en ligne non pas par l'éditeur, mais par l'ensemble des joueurs et des acteurs médiatiques et économiques tiers. Or le recours à ces supports "hors-jeu": bases de données, calculateurs, cartographies, sites spécialisés, etc. qui pour la plupart suivent l'actualité du jeu officiel, comme nous l'avons montré, est loin d'être négligeable, et fait part entière de la pratique du jeu pour de nombreux joueurs.

\section{Les serveurs privés comme ressource technique pour jouer avec l'échelle des collectifs de joueurs}

23 Si les serveurs privés offrent à leurs joueurs des versions de l'univers qui ne sont que légèrement altérées au regard de la version officielle, qu'est-ce qui peut représenter leur attrait?

24 Les serveurs privés de WoW se démarquent bien sûr d'abord par leur gratuité d'accès. Il semble en effet, et il ressort des entretiens, que ce point soit un élément convaincant en particulier pour des publics jeunes. Mais il ne suffit pas à expliquer l'engagement des joueurs dans ces espaces alternatifs, ainsi que l'ont relevé Debeauvais et Nardi en étudiant le phénomène des serveurs privés développés autour d'un jeu dont l'accès est gratuit. ${ }^{9}$ Les trajectoires et les discours de joueurs rencontrés et interrogés, tendent à confirmer ce point. Certes l'accès gratuit est un argument, notamment pour commencer à jouer. Mais plus que la gratuité, ce sont sans doutes les modalités de la consommation qui peuvent être percues comme différentes et apréciées par les joueurs. Le rapport aux dépenses liées à la pratique reste en effet très ambigü, car de nombreux serveurs privés monnayent un certain nombre de services, et les joueurs semblent y avoir fréquemment recours. L'une de nos enquêtées, par exemple, apprécie 
la gratuité d'accès du jeu mais relève elle-même avoir paradoxalement dépensé plus de 200 euros pour l'achat de personnages à haut niveau et de divers objets lors de la migration sur un nouveau serveur. Difficile à concevoir sur des serveurs officiels ${ }^{10}$, notamment pour des raisons d'équité des conditions de jeu, le développement de ces échanges économiques amménagés et plus interpersonnels participe sans doute du développement de rapports sociaux singuliers autour de la pratique des serveurs privés.

25 Les joueurs que nous avons interrogés ont peu fréquenté les serveurs officiels, et insistent en fait en particulier sur la caractéristique de petit monde que forme la population de leur serveur privé. Comme souvent, c'est l'attachement à des relations en jeu qui est évoqué pour justifier leur fort engagement dans la pratique. Céline et Jean en particulier, qui ont commencé à jouer par la médiation du père de Céline et qui ont pris peu à peu des rôles de leader en organisant des raids sur leur serveur, insistent sur l'importance qu'ils accordent à l'interconnaissance, y compris avec les animateurs du jeu. Jean indique d'ailleurs sur un ton dépréciatif - à propos d'un serveur privé dont la population est particulièrement élevée, avec un millier de joueurs -- «sur ce serveur, les MJ (maitres du jeu), ils vouvoient les joueurs! ». Cette interconnaissance est soutenue par des dispositifs de communication qui n'existent pas tels quels sur les serveurs officiels, par exemple l'existence d'un chat vocal à l'échelle du serveur ${ }^{11}$ et accessible hors connexion au jeu - Céline indique ainsi parfois rester connectée sur le vocal du serveur privé pendant ses journées de travail. Ils soulignent aussi les effets de réputation qui tendent à réguler pour eux davantage sur le serveur privé que sur l'officiel les comportements des joueurs : "c'est comme un petit village de campagne, les réputations te collent à la peau » en contrepartie, ils relèvent les proportions importantes prises par les rumeurs et les « affaires » à régler.

26 L'une de ces affaires - l'accusation de l'exploitation d'un bug de leur part lors d'une instance de raid, ainsi que la diminution de la fréquentation du serveur -- est néanmoins ce qui les pousse à migrer vers un autre serveur, ce qui n'est pas sans conséquence sur leur pratique du jeu, dont le rythme s'est espacé par la suite. La migration en cours est en effet vécue comme nécessaire plus que volontaire et n'est d'ailleurs pas pour eux anodine: "tu débarques et tu te rends compte que tu dois tout refaire, t'es un peu personne, tu connais personne, tu as tout à prouver de nouveau ... et en plus, tu te rends compte qu'ils sont moins bons que toi ». Ce dernier point laisse encore une fois à penser que si les serveurs proposent en théorie des répliques identiques du jeu, ils ne sont pas tout à fait interchangeables dans la pratique des joueurs.

\section{Conclusion : faire bande à part ?}

L'échelle de la population des joueurs semble bien être un facteur prédominant dans l'existence et l'évolution des serveurs privés que nous avons observés. Ils permettent aux joueurs non pas de jouer à un autre jeu, mais bien de faire "bande à part", en recréant les conditions de la création et du maintien d'un entre-soi que l'ouverture de l'audience des jeux en ligne, dans le cas de World of Warcraft, avait rendu plus complexe. L'effet de petit monde semble particulièrement efficace ici parce qu'il laisse de l'espace pour le développement d'une expérience qui - bien qu'inscrite dans l'expérience partagée par plusieurs millions de joueurs de World of Warcraft - peut se développer comme singulière pour un collectif. Le phénomène des serveurs privés semble tout à 
fait articulé à ces logiques sociales qui sont à la fois inscrites dans des pratiques culturelles globalisées et ancrées dans des espaces techniques localement adaptés, ce que souligne d'une certaine manière Celia Pearce lorsqu'elle se penche sur la question des migration de communautés de joueurs d'un monde à un autre: "Studying these transwolrd migratory patterns provides a glimpse of how the design of networked virtual worlds affects the emergent behaviors that happen within and through them.» (Pearce 2009). Pour le dire autrement, par ma pratique de WoW sur les serveurs officiels, je partage un ensemble de références et, d'une certaine manière, une expérience commune de certains espaces du jeu avec les joueurs des serveurs privés. Ce que je ne partage pas avec eux, c'est l'encastrement de cette expérience dans l'histoire singulière de leur serveur privé, qui est intrinsèquement articulée à l'échelle du monde, à ses particularités techniques (bugs, etc.) à ses supports d'interaction, à ses relations avec l'écosystème des serveurs francophones privés, etc. Les migrations et les déplacements d'un serveur à un autre, comme le jeu avec l'échelle du monde que proposent les serveurs prixés de WoW sont une occasion d'expérimenter de manière sensible le caractère très local de nos expériences d'un même monde de jeu.

L'investigation sur le phénomène des serveurs privés reste à développer, la question notamment de leur articulation avec les serveurs officiels, du point de vue de la technique comme de la pratique du jeu mériterait d'être davantage interrogée. Il en va de même de l'homogénéité culturelle et sociale des populations de joueurs de ces espaces. Cette trop courte exploration permet cependant d'ores et déjà de contribuer à une réflexion sur le fonctionnement et les enjeux des infrastructures informationnelles contemporaines, et d'approfondir la question de l'articulation des échelles globales et locales concernant des formes de sociabilités médiatisées. Elle met en effet en lumière la manière dont les joueurs développent et adhèrent à ces serveurs altervatifs sur des logiques de localisme, tout en soulignant le fait que ces logiques restent cadrées par un univers de jeu dont la structure générale est partagée à l'échelle globale avec un ensemble plus large de joueurs. Autrement dit, elles ne remettent en aucun cas en question l'autorité exercée par l'éditeur sur le monde de jeu.

Reste encore à mieux définir ce que des logiques « locales » représentent dans le cas d'univers fictionnels en ligne, et à continuer d'interroger l'articulation des dimensions techniques et sociales de ces phénomènes. Au-delà du rapport au jeu lui-même en tant qu'environnement, les joueurs affichent et déclarent en effet fréquemment un attachement important à des collectifs en particulier, décrits comme des " communautés ». On ne peut cependant détacher l'appréhension de ces collectifs, leurs fonctionnements singuliers, des espaces techniques, culturels et médiatiques dans lesquels ils se développent. D'une certaine manière, ces constats posent la question de la possibilité de migration des collectifs de joueurs d'un monde à un autre : dans quelle mesure les agencements sociotechniques de nouveaux espaces leur permettent-ils de faire persister un monde et une culture de jeu? 


\section{BIBLIOGRAPHIE}

BARTLE Richard (2004). Designing Virtual Worlds, Indianapolis, New Riders Pub.

BERRY Vincent (2012). L'expérience virtuelle. Jouer, vivre, apprendre dans un jeu vidéo, Rennes, Presses Universitaires de Rennes.

BLANCHET Alexis (2010). Des pixels à Hollywood: cinéma et jeu vidéo, une histoire économique et culturelle, Châtillon, Pix'n love.

BOELLSTORFF Tom (2008). Coming of Age in Second Life: An Anthropologist Explores the Virtually Human, Princeton, Princeton University Press.

BOUTET Manuel (2010). «Innovation par l'usage et objet-frontière. Les modifications de l'interface du jeu en ligne Mountyhall par ses participants ", Revue d'anthropologie des connaissances 4 (1), pp. 87-113.

BOWKER Geoffrey C., BAKER Karen, MILLERAND Florence \& RIBES David (2009). « Toward Information Infrastructure Studies: Ways of Knowing in a Networked Environment », in Hunsinger Jeremy, Klastrup Lisbeth \& Allen Matthew (dir.) International Handbook of Internet Research, Dordrecht, Springer, pp. 97-117.

CAIRA Olivier (2007). Jeux de rôle : les forges de la fiction, Paris, CNRS Éditions.

CASSELL Justine \& JENKINS Henry (2000). From Barbie to Mortal Kombat: Gender and Computer Games, Cambridge, MIT Press.

COAvoux Samuel (2010a). « La carrière des joueurs de World of Warcraft », in Craipeau Sylvie, Genvo Sébastien \& Simonnot Brigitte (dir.) Les jeux vidéo au croisement du social, de l'art et de la culture, Nancy, Presses universitaires de Nancy, pp. 43-58.

COAvoux Samuel (2010b). «L'espace social des pratiques de World of Warcraft », in Ter Minassian Hovig \& Rufat Samuel (dir.) Les jeux vidéo comme objet de recherche, Paris, Questions théoriques, pp. 164-180.

CORNELIUSSEN Hilde \& WALKE RETTBERG Jill (2008). Digital Culture, Play, and Identity: A World of Warcraft Reader. Cambridge, MIT Press.

DAYAN Daniel (1986). « Copyrighted Subcultures », American Journal of Sociology, 91, pp. 1219-1228. DEBEAUVAIS Thomas \& NARDI Bonnie (2010). « A Qualitative Study of Ragnarök Online Private Servers: In-Game Sociological Issues ", Proceedings of the Fifth International Conference on the Foundations of Digital Games, pp. 48-55 [en ligne], consulté 10.12.2015. URL : http:// www.artifex.org/ bonnie/Debeauvais-Nardi-RO-private-servers.pdf.

DE PAOLI Stefano \& KERR Aphra (2012). «L'agencement de la triche. Aborder la triche dans les MMORPG comme un imbroglio », Réseaux, 3-4 (173-174), pp. 235-269.

DiBbell Julian (1998). My Tiny Life: Crime and Passion in a Virtual World, New York, Holt.

FINE Gary Alan (2015). « Organiser les mondes de loisir : la mobilisation des ressources », Tracés, 28, pp. 157-182 [1989]. GOFFMAN Erving (1961). Fun In Games, Indianapolis, Bobbs-Merrill. JENKINS Henry (2006). Fans, Bloggers, and Gamers : Exploring Participatory Culture, New York, New York University Press. 
JOUËT Josiane (1989). « Une communauté télématique : les Axiens », Réseaux, 7 (38), pp. 49-66. KLINE Stephen, DYER-WITHEFORD Nick \& DE PEUTER Greig (2003). Digital Games: The Interaction of Technology, Culture, and Marketing, Montréal, McGill-Queen's University Press.

MALABY Thomas M. (2009). Making Virtual Worlds: Linden Lab and Second Life, Ithaca, Cornell University Press.

MAUCo Olivier (2011). « À la croisée des mondes : le game design de la sociabilité dans les Massive Multiplayer Online Games », in Granjon Fabien \& Denouël Julie (dir.) Communiquer à l'ère numérique : regards croisés sur la sociologie des usages, Paris, Presses des Mines, pp. 283-314

PEARCE Celia (2009). Communities of Play: Emergent Cultures in Multiplayer Games and Virtual Worlds, Cambridge, MIT Press.

RHEINGOLD Howard (2000). The Virtual Community: Homesteading on the Electronic Frontier, Cambridge, MIT Press.

RUEFF Julien (2010). « Reconnaissance 2.0 : controverse autour de la mise en visibilité des minorités sexuelles dans World of Warcraft », Proulx Serge, Millerand Florence \& Rueff Julien (dir.) Web social : mutation de la communication, Québec, Presses de l'Université du Québec, pp. 205215.

SHAW Adrienne (2011). « Do You Identify as a Gamer? Gender, Race, Sexuality, and Gamer Identity ", New Media \& Society, 14 (1), pp. 28-44.

HALl Stuart (1994). « Codage/Décodage », Réseaux, 12 (68), pp. 27-40 [1973].

TAYLOR T.L (2006a). « Whose Game Is This Anyway? », in Taylor T.L, In Play between Worlds: Exploring Online Game Culture, Cambridge, MIT Press, pp. 125-150

TAYLOR T. L. (2006b). « Does WoW Change Everything? How a PvP Server, Multinational Player Base, and Surveillance Mod Scene Caused Me Pause », Games and Culture, 1 (4), pp. 318-337. VÉTEL Bruno (2013). « Déviance en ligne. Enquête sur les serveurs illégaux de jeux vidéos », Terrains \& Travaux, 22, pp. 115-134.

WILlIAMS, Dmitri, YeE Nick \& CAPLAN Scott E. (2008). « Who Plays, How Much, and Why? Debunking the Stereotypical Gamer Profile », Journal of Computer-Mediated Communication, 13 (4), pp. $993-$ 1018 .

ZABBAN Vinciane (2009). « Hors Jeu ? Itinéraires et espaces de la pratique des jeux vidéo en ligne », Terrains \& Travaux, 15 (143), pp. 81-104.

ZABBAN Vinciane (2011a). « Un monde en partage. La socialisation des espaces médiatiques du jeu en ligne, ou les reconfigurations sociotechniques du monde ludique », in Perraton Charles, Fusaro Magda \& Bonenfant Maude (dir.) Socialisation et communication dans les jeux vidéo, Montréal, Presses de l'Université de Montréal, pp. 145-164.

ZABBAN Vinciane (2011b). «Ceci Est Un Monde. » Le partage des jeux en ligne : conception, techniques et pratiques, Thèse de doctorat de sociologie, Université Paris-Est Marne la Vallée.

\section{NOTES}

1. Il ne s'agit pas ici de faire une histoire des mondes de jeux en ligne, mais on peut signaler qu'à cette période se développent aussi des titres plus expérimentaux en termes d'univers thématiques tels que Mankind (Vibes Online Gaming 1998), Anarchy Online (Funcom 2001), etc. 
2. Entretien mené dans le cadre d'un terain portant sur la conception, les relations et médiations entre concepteurs et joueurs (Zabban 2011b).

3. Cf. les propos rapporté par Jessica Mulligan dans Designing Virtual World (Bartle 2004) concernant l'importance des revenus générés par les jeux en ligne sur des réseaux informatiques pionniers tels que Quantum Link ou Genie.

4. Ce qu'on peut observer dans les récits tels que ceux de Dibbel ou Rheingold concernant la formation de "communauté virtuelles" (Dibbell 1998; Rheingold 2000), et même dans une certaine mesure dans l'analyse qu'offre Josiane Jouët de la communauté télématique des Axiens, où l'anonymat n'exclue pas une logique élitaire (Jouet 1989).

5. WoW autorise le développement de modifications d'interface sur les logiciels "client" des joueurs (qui n'altèrent pas le fonctionnement du jeu). L'usage de ces modifications d'interface est parfois conséquent, et s'avère souvent essentiel à la pratique des joueurs. C'est le cas notamment des modifications qui servent de support à la coordination dans les actions collectives.

6. Un récit à prétention historique du développement d'une communauté et de technique autour des émulateurs de serveurs privés est disponible ici : http://www.gotwow.ic.cz/ancient-history/.

7. Le terme de négociation n'est cependant pas tout à fait satisfaisant, dans la mesure où les serveurs privés se développent précisemment à la marge de l'arène de négociation de la définition du monde de jeu.

8. L'expression vanilla fait a priori référence au caractère « basique » de ce parfum.

9. La version officielle du jeu qu'étudient les auteurs, Ragnarok Online propose en effet un accès gratuit, et pourtant un nombre important de serveurs privés ont été développés en marge du jeu, et représentent au final une proportion non négligeable de la totalité des joueurs.

10. Blizzard propose également pour WoW des services payant, mais est longtemps resté très attentif à ne pas introduire d'éléments qui puisse par un facteur externe au jeu (de l'argent réel) renverser directement les chances de progression des joueurs.

11. L'usage de serveurs de discussion vocale est très répandu chez les joueurs y compris du jeu officiel car elle permet une coordination plus efficace, mais est prise en charge de manière individuelle, or du contrôle de l'éditeur, et en général à l'échelle d'une ou de plusieurs guildes et non de serveurs dans leur ensemble.

\section{RÉSUMÉS}

Les jeux en ligne ont connu depuis les années 2000, parallèlement à la diffusion des usages d'Internet, un accroissement et une diversification conséquente de leur audience. Le succès de World of Warcraft (WoW) a ainsi souvent été décrit comme un phénomène participant d'une sorte "globalisation culturelle» de ce type de divertissement. Partant de travaux antérieurs concernant l'usage et la régulation de ces univers de jeu, ce texte s'appuie sur une enquête exploratoire pour interroger la pratique de WoW sur des serveurs privés, soit à la marge de la version officielle. L'exploration amène à souligner, plus qu'un désir de contestation des choix des concepteurs ou de réinterprétation du monde de jeu, le goût chez les joueurs rencontrés pour la plus petite échelle d'interaction que ces espaces présentent. Jouer avec l'échelle du monde de jeu favorise ainsi la formation d'entre-soi qui se développent dans espaces techniques locaux, mais restent ancrés dans l'infrastructure globale du jeu. La pratique du jeu sur des serveurs privés renvoie alors à un questionnement plus large vers la complexité avec laquelle s'articulent logiques locales et globales au travers des médiations numériques. 
In parallel with the diffusion of Internet use, online games have known since the 2000's a consequent growth and diversification of their audience. The success of World of Warcraft (WoW) has often been described as a phenomenon involved in a kind of "cultural globalization" of this type of entertainment. Grounded on a previous work concerning the use and regulation of the game world, and an exploratory survey, this text investigates the practice of WoW on private servers, at the margins of the official version. More than a desire for challenging game designers or of reinterpretating of the game world, the exploration leads to stress the interest that players show in this places because of the world small scale of interaction they provide. Playing with the scale of the game as a social world thus favors the emergence of forms of elective communities which develop in technically local spaces, but are staying anchored in the global game infrastructure. The practices of private servers practices thereby refer to wider questions regarding the complexity with which local and global logics are articulated through mediations digital.

INDEX

Mots-clés : jeu en ligne, serveurs privés, régulation, entre-soi, global/local, infrastructure

Keywords : online game, private servers, regulation, elective communities, global/local, infrastructure

\section{AUTEUR \\ VINCIANE ZABBAN}

Université Paris 13, EXPERICE 